\title{
Enfoque STEAM como mejora en el proceso de enseñanza-aprendizaje braille para deficientes visuales en Cuenca
}

\section{STEAM approach as an improvement in the braille teaching/learning process for the visually impaired in Cuenca.}

\author{
Mateo Elian Buestán Abril. ${ }^{1}$, Henry David Curillo Suin. ${ }^{2}$ \& Galo Patricio Hurtado \\ Crespo. ${ }^{3}$
}

\begin{abstract}
In the city of Cuenca, Ecuador, the pandemic has hit hard affecting the main areas: economic, political and social. As a consequence, several vulnerable groups of the population have been forced to change their way of life, the population group that is the focus of this work are people with visual impairment. They manifest a degree of impairment to a greater or lesser extent to the perception of light in the ocular system, causing them not to distinguish clearly objects around them, hindering their quality of life. As a consequence, their other senses, for example, touch and hearing, become more acute. In order to be included in the social and work environment, they must learn a means of communication that allows them to perform autonomously with society, for this reason, they learn the Braille system, which consists of six dots located in two columns and three rows, Braille integrates letters, numbers and signs. Learning this system is complex at the beginning, even more so using traditional methods such as the ruler and the stylus. Objectives: To design an electronic device to improve the Braille teaching-learning system for people with visual disabilities. Methodology: The descriptive methodology is applied with a quantitative approach, non-experimental type and to obtain greater precision in the results, it is used longitudinally, to facilitate the adaptation of the individual with the technological device, STEAM is integrated to the teaching-learning process. Contributions: This article aims to achieve as a result that people with visual impairment not only learn letters and numbers, but also acquire more autonomous skills
\end{abstract}

\footnotetext{
${ }^{1}$ Instituto Tecnológico Superior Sudamericano, Cuenca, Ecuador, mebuestan@sudamericano.edu.ec

${ }^{2}$ Instituto Tecnológico Superior Sudamericano, Cuenca, Ecuador, hdcurillo@sudamericano.edu.ec

${ }^{3}$ Instituto Tecnológico Superior Sudamericano, Cuenca, Ecuador, gphurtado@sudamericano.edu.ec
} 
in their instruction and thus in the future the project would benefit the inclusion in different areas of society.

Keywords: braille, disability, learning, visual, device, teaching, electronic, hardware.

\section{Resumen}

En la ciudad de Cuenca, Ecuador, la pandemia golpeo fuertemente afectando los ámbitos principales: económicos, políticos y sociales. Como consecuencia diversos grupos vulnerables de la población se han visto obligados a cambiar su forma de vida, el conjunto de población en el que se centra este trabajo son las personas con discapacidad visual. Ellos manifiestan un grado de deficiencia en mayor o menor medida a la percepción de luz en el sistema ocular, provocando que no distingan de manera clara objetos a su alrededor, dificultando su calidad de vida. Como consecuencia, agudizan sus otros sentidos, por ejemplo, el tacto y el auditivo. Para incluirse en el ámbito social y laboral deben aprender un medio de comunicación que les permita desempeñarse de forma autónoma con la sociedad, por tal motivo, aprenden el sistema braille que consta de seis puntos ubicados en dos columnas y tres filas, el braille integra letras, números y signos. Aprender este sistema se torna complejo al inicio, utilizando métodos tradicionales como la regleta y el punzón. Objetivos: diseñar un dispositivo electrónico que permita mejorar el sistema de enseñanza-aprendizaje braille en personas con discapacidades visuales. Metodología: se aplica la metodología descriptiva con enfoque cuantitativo, de tipo no experimental y para obtener mayor precisión en los resultados se ocupa de forma longitudinal, para facilitar la adaptación del individuo con el dispositivo tecnológico se integra STEAM al proceso de enseñanza-aprendizaje. Aportes: el presente artículo pretende conseguir como resultado que las personas en situación de discapacidad visual no solo aprendan letras y números, sino que adquieran habilidades más autónomas en su instrucción y de esta manera a futuro el proyecto beneficiaría a la inclusión en diferentes ámbitos de la sociedad.

Palabras claves: braille, discapacidad, aprendizaje, visual, dispositivo, enseñanza, electrónico, hardware.

\section{Introducción}

Según el decreto número 17 de la Ley Orgánica de Discapacidades del Ecuador, reconoce, ejerce y garantiza los derechos propios de las personas en condición humana de vulnerabilidad en la que se encuentre la persona con discapacidad. Las personas en situación de discapacidad son un grupo vulnerable de la población, porque su condición ha dificultado su desarrollo integral, limitando su aprendizaje normal y la adquisición de habilidades básicas como la lectura, escritura, comunicación y el acceso a información. Teniendo en cuenta que el aprendizaje braille inicia desde una etapa temprana entre aquellas personas que nacieron no videntes o con el transcurso del tiempo sufrieron un déficit visual siendo mayores, resultado de una alteración en su sistema visual o por un 
accidente severo. Sin embargo, al perder el sentido de la vista, este es sustituido por varios sentidos, el más común es el sentido del tacto, convirtiéndose en la puerta de comunicación con el mundo. De esta manera, el siguiente estudio tiene como objetivo determinar a través de una revisión teórica, diversos factores que influyen en el proceso de enseñanza braille, para establecer un dispositivo que permita incrementar el nivel de aprendizaje en personas que se encuentren en situación de discapacidad visual y también entre aquellas que no padezcan ningún déficit visual, pero desean tener conocimiento del sistema de comunicación braille. Es por ello que el dispositivo a desarrollar ha tomado como base la experiencia de personas no videntes y sus necesidades, con su ayuda se busca darle características de fácil manejo. El trabajo presenta la siguiente estructura: Se presenta la modalidad de investigación apropiada para el desarrollo del proyecto y el procedimiento adecuado para implementar la metodología. Se describe el desarrollo del dispositivo electrónico, considerando los elementos que intervienen en su implementación, como herramientas de hardware como software. El software se basa en que el Arduino (2020) recibe los pulsos del dispositivo, de acuerdo a las combinaciones existentes en el signo generador ( 6 puntos 18 distribuidos en dos columnas y tres filas) para formar cada letra o número. El tamaño y distribución de los 6 puntos forman el llamado Signo Generador. "Las terminaciones nerviosas de la yema del dedo captan este tamaño" (Gómez Viñas, 2004). Utilizaremos este sistema para desarrollar un dispositivo, que permita a las personas con discapacidad visual aprender un medio de comunicación alternativo para que puedan ser autónomos, tal vez no en su totalidad, pero sí en su mayoría. Según la afirmación propuesta en 1948, por La Declaración Universal de los Derechos Humanos, se dicta de forma clara: "Todos tienen derecho a la educación”, debe ser gratuita y de manera obligatoria al menos el nivel primario (Crosso, 2010). La Federación Nacional de Ciegos del Ecuador (FENCE), es una institución con derecho jurídico que busca el desarrollo de las personas no videntes a través de programas que garanticen la mejora en su calidad de vida, entre ellas está la alfabetización, inserción laboral (FENCE, 2017). El artículo 47 de la Ley Orgánica de Educación Intercultural del Ecuador, se cita que: "El Estado ecuatoriano garantizará la inclusión e integración de estas personas en los establecimientos educativos" (Asamblea Nacional, 2017). Sin embargo, esta propuesta no se cumple, debido a la falta de presupuesto en centros educativos con instalaciones adecuadas o la limitada formación de docentes en educación especial a través del sistema braille (Aldaz, 2016). Una vez realizados los objetivos planteados del proyecto se procede a redactar las conclusiones y recomendaciones. Durante el desarrollo del proyecto, surgió la necesidad de investigar a profundidad el funcionamiento del sistema braille, su sintaxis, combinaciones, su aplicación en la vida empírica de una persona no vidente, la cantidad de personas con discapacidad visual en la provincia del Azuay y concretamente en el cantón Cuenca a través de los cortes de registro del CONADIS (Consejo Nacional para la Igualdad de Discapacidades , 2020). A futuro, implementar el proyecto podría potenciar la inclusión social de tal forma que el dispositivo beneficie a las personas que lo necesiten, así cualquier persona que lo adquiera podrá continuar con su aprendizaje. 
Marco Teórico

Mediante una investigación exploratoria, definimos los tipos de discapacidades existentes y su limitación a ciertas actividades: Discapacidad Motriz (MIES, 2013), Sensorial, Intelectual (Morales et al., 2015) y Visual. Del mismo modo se identificó los diferentes grupos de Personas no Videntes como: Personas con Deficiencia Visual (Puntodis, 2016) y Personas con Ceguera Total. Referente a los métodos para el proceso de enseñanzaaprendizaje braille se investigaron algunos procedimientos en distintas edades:

\section{Bliseo}

Este método de aprendizaje es dedicado a las personas adultas que son alfabetizados, se empieza profundizando en todo lo relacionado con el signo generador, para después poco a poco ir insertando las letras y números en pequeñas series hasta abarcar todas las combinaciones (Martínez \& Polo, 2004).

\section{Pérgamo}

Es un método de alfabetización para personas adultas no videntes. Se comienza con una serie de ejercicios con el objetivo de identificar la posición de los puntos en el cajetín. El orden en que se enseña las letras del alfabeto es diferente, primero van las vocales (a, e, i, o, u) y luego las demás letras así. Una vez terminado las letras en minúsculas, se aprende en mayúsculas y para finalizar el punto y la coma (Martínez \& Polo, 2004). Al final se incluyen aquellas letras que se ocupan menos (x, q, ch, k, w, ü).

\section{Tornillo}

Lo distinto de este método es que va dirigido a la población para iniciarlos en la lectura braille, sin embargo, también se adapta a cualquier edad que se necesite, explora el desarrollo del tacto. Se emplea material didáctico estimulante a través de representaciones en relieve, se utilizan cuentos con frases y párrafos con sentido. Se empieza con aquellas letras más fáciles de distinguir al tacto hasta que se abarca todo el abecedario para luego ir introduciendo poco a poco las vocales con tilde.

Como se puede observar en este método influyen diversos factores como "la motivación, la cantidad de estímulos lectores que recibe el niño, la edad de comienzo de la lectura"(Martínez \& Polo, 2004), por lo que se debe elegir lo más conveniente para el infante dependiendo su caso.

\section{Punto a Punto}

Está en dos idiomas, el catalán y el castellano, a diferencia de las anteriores, este método consta de dos series, la primera abarca puntos para la prelectura y preescritura, mientras que la segunda serie se centra en la enseñanza del sistema braille (Martínez \& Polo, 2004).

La primera serie: Presenta ejercicios para el reconocimiento de objetos según su forma, tamaño, también da un seguimiento de orientación, para así llegar al final de esta primera 
parte donde se empieza con la introducción al sistema braille, aprendiendo primero del signo generador.

La segunda serie: Se va presentando y aprendiendo poco a poco cada letra del abecedario, se realizan ejercicios para mejorar y controlar el sentido del tacto, se empieza a combinar las letras para formar palabras, oraciones. La metodología se respalda con dibujos en relieve para motivar al alumno.

\section{SCRUM}

Según Rodríguez y Dorado (2015) en el año 1986, aparece una nueva forma de gestionar los proyectos con mayor agilidad y flexibilidad lo que se denomina hoy en día como SCRUM. Es una metodología de desarrollo ágil que tiene como principio crear ciclos pequeños para su desarrollo y a lo largo del proyecto se va progresivamente aumentando el resto de funcionalidades.

En 1996, se presentaron por primera vez buenas prácticas para el proceso de desarrollo de productos en diversos entornos que resultó adecuado para las empresas. En la actualidad SCRUM es una de las metodologías ágiles más usadas y fuertes no solo en el campo de software sino también en el campo de la producción, educación y comunicación.

\section{STEAM}

En Estados Unidos en la década de los 90s, la Fundación Nacional para la Ciencia en Estados Unidos (NFS), introduce por primera vez el término STEM, que buscaba impulsar la investigación científica a la par de la tecnología. Sin embargo, esta idea tiene su auge en la década de los 2000s, integrando la educación artística, dando origen al acrónimo de STEAM, nombrado por Yakman en el 2008.

STEAM involucra el aprendizaje de cinco disciplinas que se integran entre sí para buscar soluciones en distintas áreas a la vez. STEAM son las iniciales en inglés de las disciplinas que lo forman: Ciencia, Tecnología, Ingeniería, Arte y Matemáticas. Según Yakman (2008) la metodología STEAM, brinda un desarrollo de un modelo educativo que une las diversas materias académicas y cambie la forma tradicional de enseñanza a algo más práctico y teórico a la vez.

\section{Metodología}

La metodología empleada es de tipo descriptiva con un enfoque cuantitativo. Se revisaron artículos en base de datos científicas como: Google académico, Dialnet. Por medio de una revisión sistemática se extrajeron tendencias tecnológicas y métodos aplicados al proceso enseñanza-aprendizaje braille en las personas con discapacidad visual. La investigación descriptiva permite recolectar datos sobre diferentes aspectos del usuario, para realizar un análisis profundo encontrando patrones, comportamientos, actitudes y medir su tendencia de aprendizaje a lo largo del tiempo. El estudio descriptivo mide las variables de manera independiente y con mayor precisión utilizando tres métodos distintos: La entrevista, 
encuestas y la observación. El método observacional es el más eficaz, permite registrar el comportamiento del individuo de manera natural, facilita la recopilación objetiva de datos en valores numéricos.

La investigación es de tipo no experimental, porque no se pretende manipular las variables, los datos obtenidos serán analizados tal y como se obtiene directamente de los individuos participantes. Se limita únicamente a observar situaciones existentes en un contexto natural. La investigación no experimental puede ser de dos tipos: transversal o longitudinal, para beneficio del artículo científico se elige la investigación longitudinal, faculta a los investigadores analizar los cambios que ocurren entre las variables a lo largo del tiempo, es decir, se estudia la tendencia del grupo de muestra.

La metodología STEAM facilita la adaptación del individuo con un dispositivo tecnológico, debido que es un modelo que promueve la integración de materias científicotécnicas y artísticas, según Yakman (2008) STEAM plantea que el aprendizaje sea de manera integrada con dos enfoques teórico y práctico. La metodología trabaja los problemas en diferentes disciplinas para obtener soluciones creativas y a la vez innovadoras, aprovechando en lo mayor posible las tecnologías existentes para mejorar las capacidades del individuo.

Su propósito es muy beneficioso porque permite alcanzar las metas de aprendizaje de una manera efectiva, eficiente y eficaz, aprovecha las capacidades de los estudiantes y docentes, recompensa el tiempo que cada individuo dedica a su aprendizaje. En consecuencia provoca un impacto al interés de la tecnología y sus materias, lo que vuelve a STEAM adaptable a diversos escenarios educativos y apoya en gran medida el proceso de enseñanza-aprendizaje utilizando tiempo, recursos e infraestructuras.

\section{Desarrollo}

Se realiza el diagrama de flujo del funcionamiento del dispositivo electrónico, para detallar los procesos de funcionamiento que realiza según el tema planteado. En base al diagrama se identifican las conexiones necesarias que debe tener el dispositivo y el número específico de entradas y salidas de señales digitales.

Efectuado el análisis de funcionamiento y conexiones, se procede a determinar los componentes electrónicos a utilizar, dividiendo el dispositivo en módulos:

- Módulo de Lógica-Control y Comunicación.- El Arduino Nano, es el componente más importante, debido a que controla y procesa todo el funcionamiento del dispositivo. Es una placa microcontroladora que en comparación con el Arduino tradicional, es más pequeña y cuenta con un mayor número de pines de conexión.

- Módulo de Almacenamiento.- El módulo MicroSD, permite la conexión de una placa microcontroladora a una tarjeta de memoria SD. Su objetivo es almacenar 
la información, como los archivos multimedia utilizados en el dispositivo, para que el Arduino pueda acceder a los audios y reproducirlos.

- Módulo de Entrada-Salida.- Para el ingreso de datos del dispositivo se utiliza un teclado, en este caso pulsantes THT que actúan como entrada, debido a que sus dimensiones son pequeñas y permiten una mejor distribución en la placa PCB (Placa Electrónica Impresa).

- Módulo de Audio.- Una parte importante del dispositivo es ayudar al usuario a conocer que está enviando, y cómo manipular el dispositivo para su aprendizaje, por tal motivo, se decide implementar un componente sonoro (Altavoz) con el objetivo de guiar al usuario, para que conozca las combinaciones, o si desea ingresar números o letras.

- Módulo de Alimentación.- El módulo de carga es otro elemento electrónico indispensable para el funcionamiento del dispositivo, además de cargar la batería también ofrece una protección a los componentes electrónicos en caso de darse un cortocircuito, lo que se vuelve fundamental para este tipo de proyectos.

Luego de seleccionar los componentes electrónicos adecuados para el dispositivo, se procede a realizar el diagrama de conexiones del circuito electrónico. A partir del esquema, se inicia el armado del circuito en una placa de pruebas (Protoboard), para comprobar el funcionamiento correcto del dispositivo. Finalizadas las pruebas correspondientes se conduce a elaborar el diseño de la placa PCB, utilizando el software de EasyEDA, para posteriormente imprimirlo y realizar el ensamblaje correspondiente de todos los componentes electrónicos utilizados.

\section{Resultados}

Para obtener un análisis e interpretación de los resultados del dispositivo electrónico realizamos una entrevista en el centro SONVA (Sociedad No-Videntes del Azuay Ecuador), utilizando un banco de preguntas referentes a la situación actual de las personas con discapacidad visual, precios de dispositivos y capacidad de profesionales para enseñar a estas personas.

Para la entrevista se realizó una grabación a la persona notificando del tema a tratar y obteniendo su consentimiento previo. A partir de los parámetros expuestos como guía, se procede a la validación del dispositivo por parte de los expertos en el área técnica.

Los aspectos a calificar son: Aplicabilidad, Factibilidad, Innovación, Ergonómico, Fundamentación Tecnológica, Fundamentación Pedagógica, Tiempo de Aprendizaje, Indicaciones para su Uso. La escala de valores utilizada es:

- Muy Adecuado equivale a 5

- Bastante Adecuado equivale a 4

- Adecuado equivale a 3

- Poco Adecuado equivale a 2

- Inadecuado equivale a 1 
Obteniendo los resultados visualizados en la gráfica.

Figura 1

Aplicación del dispositivo braille

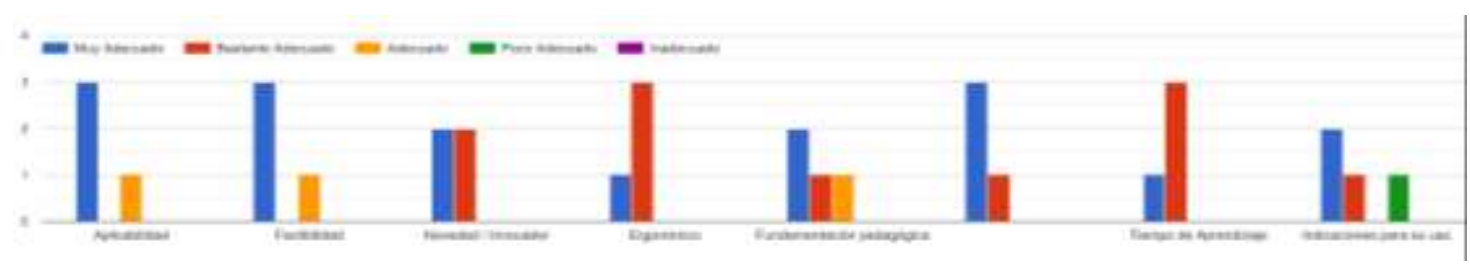

Nota. Indicadores evaluados para el funcionamiento del dispositivo.

Según los resultados obtenidos de la gráfica, podemos concluir que el dispositivo es aplicable en distintos ámbitos de enseñanza-aprendizaje braille para personas de cualquier edad. Es factible adquirir el dispositivo gracias a su bajo costo de fabricación y distribución en comparación con dispositivos existentes en el mercado. Es bastante innovador debido a su tamaño que facilita su portabilidad. Su manipulación ergonómica permite que el usuario no se canse de utilizar el dispositivo por mucho tiempo. Con respecto a la fundamentación pedagógica y tecnológica es adecuada para integrar la tecnología en el ámbito educativo. La curva de aprendizaje demuestra que tan rápido se puede aprender y comprender el lenguaje braille, esto gracias a su correcta funcionalidad. Como último punto, las indicaciones para su uso deben mejorarse a fin de que la interactividad del usuario con el dispositivo sea más óptima y eficaz. De este modo se incrementa la eficacia de la curva de enseñanza-aprendizaje braille.

Si bien la innovación tecnológica es la primera opción para impulsar este tipo de metodologías de enseñanza-aprendizaje braille, en la actualidad es difícil implementar cuando se trata de sectores rurales. Se debe tomar en cuenta el costo del dispositivo considerando los componentes electrónicos estrictamente necesarios, para lograr adaptarse a la capacidad financiera de los usuarios. Es importante agregar nuevas funcionalidades por ejemplo la capacidad de que el usuario pueda escribir textos, logrando que el dispositivo sea más completo.

\section{Conclusiones}

- La información teórica adquirida sobre los dispositivos y metodologías existentes para la enseñanza braille, permitieron solventar la necesidad de requerimientos para el diseño y construcción de un dispositivo que permita facilitar el aprendizaje en personas con discapacidad visual.

- El uso de SCRUM como metodología de desarrollo facilita el trabajo en equipo, permite realizar cambios con agilidad y gestiona cada proceso del desarrollo y su implementación es muy sencilla, haciendo que sea una metodología muy fuerte en la actualidad para diversos proyectos.

- Los parámetros de diseño establecidos en el ámbito mecánico y electrónico, permitieron construir un dispositivo con las características necesarias que faciliten la familiarización del alfabeto braille con otros dispositivos similares, alcanzando 
todas las expectativas y funcionalidades requeridas para usuarios con este tipo de deficiencia.

\section{Referencias bibliográficas}

Aldaz, A. (2016). SISTEMA ELECTRÓNICO PARA LA ENSEÑANZA DEL LENGUAJE BRAILLE A PERSONAS INVIDENTES.

Arduino. (2020). ¿Qué es Arduino? https://www.arduino.cc/en/Guide/Introduction\#whyarduino

Asamblea Nacional. (2017). Ley Orgánica de Educación Intercultural (LOEI). Ecuador, Ministerio De Educación, 417, 1-85. https://bit.ly/3hB7t8h

CONADIS. (2020). Estadísticas de Discapacidad - Consejo Nacional para la Igualdad de Discapacidades. Ministerio de Salud Pública. https://www.consejodiscapacidades.gob.ec/estadisticas-de-discapacidad/

Crosso, C. (2010). El Derecho a la Educación de Personas con Discapacidad : impulsando el concepto de Educación Inclusiva. Revista Latinoamericana de Educación Inclusiva, 4, 79-95.

FENCE. (2017). Federación Nacional de Ciegos del Ecuador. http://fenceecuador.org/

Gómez Viñas, P. (2004). La educación de las personas sordociegas: diferencias y proceso de mediación. In La sordoceguera: un análisis multidisciplinar (pp. 309-362). http://dialnet.unirioja.es/servlet/extart?codigo $=1155436$

Loza Peñaloza, O. P. (2007). Sistema electrónico braille para la ayuda en el aprendizaje $\begin{array}{lllll}\text { de personas no videntes. } & \text { Universitas, }\end{array}$ https://doi.org/10.17163/uni.n7.2006.08

MIES. (2013). Propuesta de Atención Integral para Personas con Discapacidad. 1-42.

Martínez, I., \& Polo, D. (2004). Versión para imprimir - Guía didáctica para la lectoescritura braille (SID). Madrid: ONCE, Dirección de Educación, 2004. https://sid.usal.es/version-imprimir/libros/discapacidad/12071/8-1/guia-didacticapara-la-lectoescritura-braille.aspx

Morales, K., Pineida, Z., Trujillo, L., \& Velásquez, I. (2015). PROPUESTA SOCIAL DE ATENCIÓN INTEGRAL PARA LAS PERSONAS CON DISCAPACIDAD DE LA INSPECCIÓN DE CAMBAO. https://hdl.handle.net/10656/4798

Rodríguez, C., \& Dorado, R. (2015). ¿ Por qué implementar Scrum? Why to implement Scrum? Pour quelles raisons mettre en place Scrum? Porque implementar Scrum? Revista Ontare, 3(1), 125-144. http://200.0.187.30/index.php/Revistao/article/view/1253/1218 
Vol. 5, N4, pág. 79- 89

Puntodis. (2016)

Discapacidad visual.

CULCyT,

51.

https://puntodis.com/featured_item/discapacidad-visual/

Yakman, G. (2008). STEAM Education: An Overview of Creating a Model of Intergrative Education. Tesis, 53(9), 1689-1699.

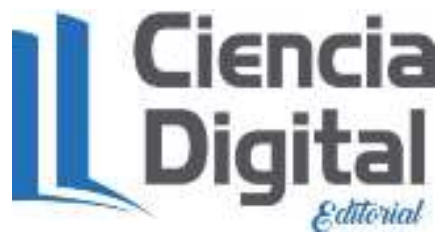




\section{PARA CITAR EL ARTÍCULO INDEXADO.}

Buestán Abril, M. E., Curillo Suin, H. D., \& Hurtado Crespo, G. P. (2021). Enfoque STEAM como mejora en el proceso de enseñanza-aprendizaje braille para deficientes $\begin{array}{lllll}\text { visuales en } \quad \text { Cuenca. } & \text { Explorador } & \text { Digital, }\end{array}$ https://doi.org/10.33262/exploradordigital.v5i4.1892

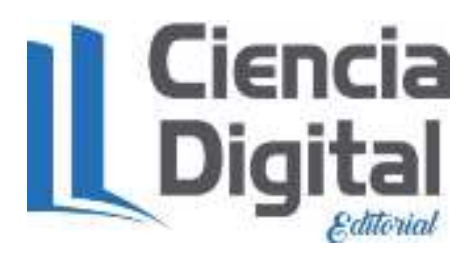

El artículo que se publica es de exclusiva responsabilidad de los autores y no necesariamente reflejan el pensamiento de la Revista Explorador Digital.

El artículo queda en propiedad de la revista y, por tanto, su publicación parcial y/o total en otro medio tiene que ser autorizado por el director de la Revista Explorador Digital.
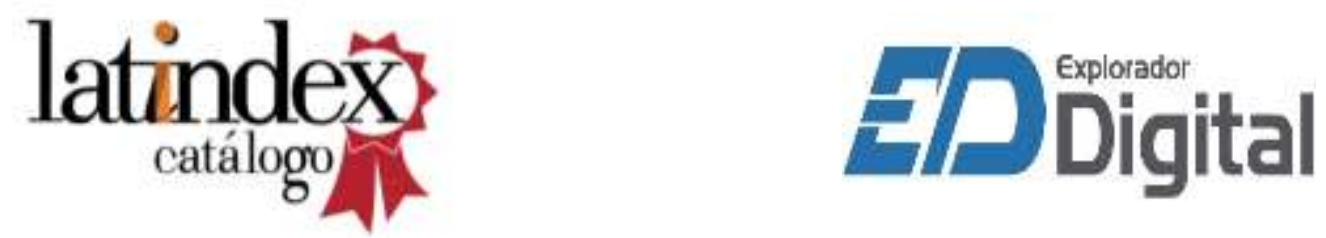